Check for updates

Cite this: RSC Adv., 2018, 8, 30978

Received 12th July 2018

Accepted 21st August 2018

DOI: $10.1039 / c 8 r a 05941 a$

rsc.li/rsc-advances

\section{A new sensitive UPLC-MS/MS method for the determination of cucurbitacin B in rat plasma: application to an absolute bioavailability study}

\author{
Ya Xiao, $\uparrow$ Qiang Zhao, $\uparrow$ Qian Wu, Jinhua Chang, Hefei Xue, Cuizhe Liu* \\ and Xigang Liu (D) *
}

Cucurbitacin $\mathrm{B}(\mathrm{CuB})$ is a highly oxygenated tetracyclic triterpene, and a Biopharmaceutics Classification System (BCS) class IV drug used for the treatment of persistent hepatitis, chronic hepatitis, and primary liver cancer. Nevertheless, CuB has low solubility and low permeability, and is present at low concentrations in the human body. The aim of this study was to develop a method for the determination of CUB in plasma using ultra-performance liquid chromatography-mass spectrometry (UPLC-MS/MS) with estrone as an internal standard (IS), as well as to examine the pharmacokinetics and absolute bioavailability of $\mathrm{CuB}$ in rats. Plasma samples were processed by liquid-liquid extraction with ethyl acetate. Separation was achieved on a BEH C18 column $(2.1 \times 50 \mathrm{~mm}, 1.7 \mu \mathrm{m})$ at $35{ }^{\circ} \mathrm{C}$ using an isocratic mobile phase system with $0.1 \%$ formic acid-acetonitrile $(50: 50, \mathrm{v} / \mathrm{v})$ at a flow rate of 0.3 $\mathrm{mL} \mathrm{min}^{-1}$. The detection was performed using a multiple reaction monitoring mode via a positive electrospray ionization interface. The calibration curves showed good linearity $(r=0.9998)$ within the tested concentration ranges. The lower limit of quantification for plasma was $0.05 \mathrm{ng} \mathrm{mL}^{-1}$; the matrix effect of CuB and IS was $94.19-99.42 \%$ and $100.83 \%$, respectively. The mean extraction recoveries from plasma were $85.34-90.53 \%$. The intra-day and inter-day accuracies and precision deviations were within $\pm 15 \%$, which was in line with the allowable range of accuracy. In addition, the stability of the method was also verified. The absolute bioavailability of orally administered CuB in rats was $1.37 \%$. To sum up, the presented method was determined to be suitable for the quantitation of CuB in rat plasma. Also, the absolute bioavailability observed in the present study suggested that it was necessary to change the dosage form to improve bioavailability, or to improve this by other means.

\section{Introduction}

Cucurbitacins are highly oxygenated, tetracyclic triterpenes that can be isolated from members of the Cucurbitaceae family such as cucumber, watermelon, and zucchini. More than 18 kinds of cucurbitacins can be found in nature, including cucurbitacin B. ${ }^{1} \mathrm{CuB}$ is recognized as the major active ingredient which possesses a broad range of pharmacological properties such as hepatoprotection, anti-tumor effects and immune enhancement., ${ }^{2,3}$ Until now, CuB has been used for the treatment of persistent hepatitis, chronic hepatitis, and primary liver cancer, as it has significant anti-inflammatory activities and antitumor effects. ${ }^{4}$ The exact mechanism of the anti-tumor effect of $\mathrm{CuB}$ appears to be related to induction of cell apoptosis and cell cycle arrest, cleavage of cytoskeletons, and blockage of cell signaling pathways. ${ }^{5-8}$ Nevertheless, there are relatively few studies on the

Hebei Key Laboratory of Research and Development for Chinese Medicine, Chengde Medical University, Anyuan Road, Shuangqiao, Chengde, Hebei 067000, P. R. China.E-mail: liuxgmail@sina.com; liucuizhexy@163.com

$\dagger$ Both authors contributed equally to this work. precise pharmacokinetic mechanism of $\mathrm{CuB}$. So far, many chromatographic systems for the determination of $\mathrm{CuB}$ content have been developed. ${ }^{\mathbf{9} 10}$ Among these, only a few can be used for the determination of plasma samples. For example, Dou et al. examined the content of CuB using HPLC. ${ }^{11}$ In addition, Wang et al. used an HPLC-UV method for the quantification of CuB with a lower limit of quantification (LLOQ) of $1.0 \mu \mathrm{g} \mathrm{mL}$ in human serum. ${ }^{\mathbf{1 2}}$ However, these approaches offered low sensitivity and poor specificity. Bajcsik et al. determined cucurbitacin B, E, I and E-glucoside by HPLC-MS with limits of quantification of $0.05-0.42 \mathrm{ng} \mathrm{mL}^{-1}$ and analysis was by Q1 scans with negative ionization. ${ }^{13}$ The emergence of new analytical methods such as UPLC-MS/MS and sample preparation advancements have increased sensitivity and efficiency. ${ }^{\mathbf{1 4 , 1 5}}$ For example, UPLC-MS/MS has been recently used to determine the content and pharmacokinetics of $\mathrm{CuB}$ in rat plasma; the LLOQ of CuB in plasma measured by this method was $0.3 \mathrm{ng}$ $\mathrm{mL}^{-1} \cdot{ }^{16}$ Moreover, Wang et al. have adopted a combined UHPLC-MS/MS system to determine the content of $\mathrm{CuB}$ and $\mathrm{CuE}$ with limits of quantification of $1.60 \mathrm{ng} \mathrm{mL}{ }^{-1} \cdot{ }^{17}$ In previous experimental studies, a novel dual modality system i.e. UPLC- 
MS/MS was established for the quantitative analysis of CuB. Nonetheless, due to the complexity of condition optimization and the lower concentration of the sample in the body, determination of the CuB content and quality control in plasma after administration still need to be further investigated.

As a Biopharmaceutics Classification System (BCS) class four drug with low solubility and low permeability, ${ }^{18,19} \mathrm{CuB}$ is present at low concentrations in the body of humans and animals. Therefore, it is very important to develop a more sensitive and simple way to determine its exact concentration in the body. Currently, there are no studies reporting the absolute bioavailability of cucurbitacin. Hence, the goal of this research was to establish and validate a highly selective and sensitive UPLC-MS/ MS method for the determination of CuB in rat plasma, and to further explore its absolute bioavailability and its effect on plasma. Solid dispersion techniques were shown to be successful in improving the dissolution and bioavailability of poorly soluble drugs by reducing the particle size of the drug, by improving wettability and by forming amorphous particles. Accordingly, a solid dispersion of $\mathrm{CuB}$ should be further investigated.

\section{Materials and methods}

\subsection{Chemicals and reagents}

The chemical standard of CuB (purity > 96.9\%, CAS111945201301) was purchased from the National Institute for Food and Drug Control (Beijing, China); the internal standard (IS) estrone (purity > 98\%, CAS C-021-170426) was supplied by Ruifensi Biotech Co. Ltd. (Chengdu, China). Their chemical constitutions are shown in Fig. 1. Acetonitrile and methanol were of MS grade and were purchased from the United States (Fisher Chemical). MS grade formic acid was procured from Spain (Fisher Scientific). Purified water for UPLC analysis was bought from Wahaha (Gaobeidian, Hebei, China).

\subsection{Animals}

Male adult Wistar rats were obtained from Beijing Vital River Laboratory Animal Technology Co. Ltd. All the animals were housed in an environment with a temperature of $22 \pm 1{ }^{\circ} \mathrm{C}$, a relative humidity of $50 \pm 1 \%$ and a light/dark cycle of 12/12 h. Before starting the experiment, the rats randomly received standard chow and sterile water and were fasted for 12 hours before the start of the experiment.

\subsection{UPLC-MS/MS conditions}

A Waters Acquity UPLC Liquid Chromatograph included a Diode Array Detector, Sample Manager, Binary Solvent Manager and Empower 2 software. Chromatography separations were performed on an ACQUITY UPLC BEH C18 column $(2.1 \times 50 \mathrm{~mm}, 1.7 \mu \mathrm{m}$; Waters Corporation, Ireland $)$, equipped with a guard column and eluted with an isocratic mobile phase system, which was composed of acetonitrile and $0.1 \%$ formic acid $(50: 50, \mathrm{v} / \mathrm{v})$. The experimental conditions were as follows: the column temperature was kept at $35^{\circ} \mathrm{C}$, the flow rate was 0.3 $\mathrm{mL} \min ^{-1}$, the injection volume was $2 \mu \mathrm{L}$ and the total running time was $2.5 \mathrm{~min}$. The mass spectrometer was equipped with a Q-Trap® 5500 triple quadruple mass spectrometer (AB SCIEX, USA), which was operated in the positive multiple reaction monitoring (MRM) mode. The ESI source and MS/MS parameters were set as follows: ion spray voltage, $+5500 \mathrm{~V}$; curtain gas, $35 \mathrm{psi}$; temperature, $450{ }^{\circ} \mathrm{C}$; ion source gas $1,45 \mathrm{psi}$; ion source gas 2, 55 psi; the interface heater was on, and the collision gas was medium. The mass transitions were: $\mathrm{CuB}, \mathrm{m} / \mathrm{z} 581.3 \rightarrow$ 521.3 , IS, $m / z 269.2 \rightarrow 145.1$, and the mass spectra of CuB and IS are shown in Fig. 1.

\subsection{Preparation of working solutions, calibration standards and quality control (QC) samples}

Stock solutions of the IS $\left(106 \mu \mathrm{g} \mathrm{mL}^{-1}\right)$ and $\mathrm{CuB}\left(120 \mu \mathrm{g} \mathrm{mL} \mathrm{m}^{-1}\right)$ were formulated with methanol. Immediately before use, serial dilution of the stock solution with methanol provided the $\mathrm{CuB}$ working solutions. $848 \mathrm{ng} \mathrm{mL}^{-1}$ was obtained as a final concentration after diluting the IS stock solution. All the standard solutions were kept at $4{ }^{\circ} \mathrm{C}$ and were protected from light until further use.

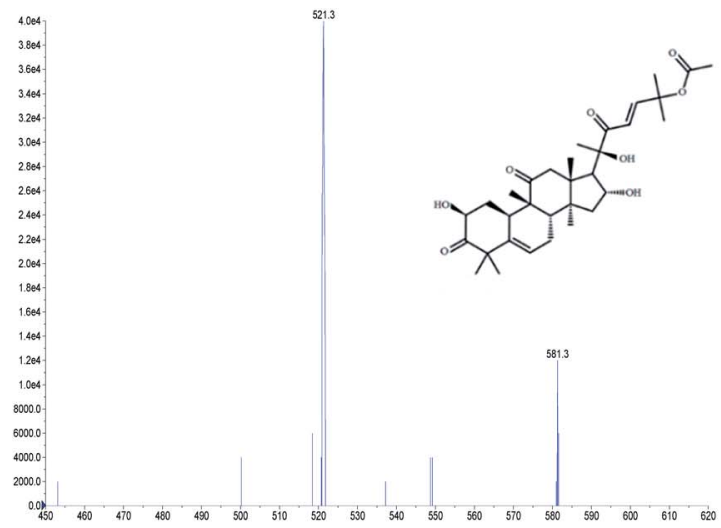

A

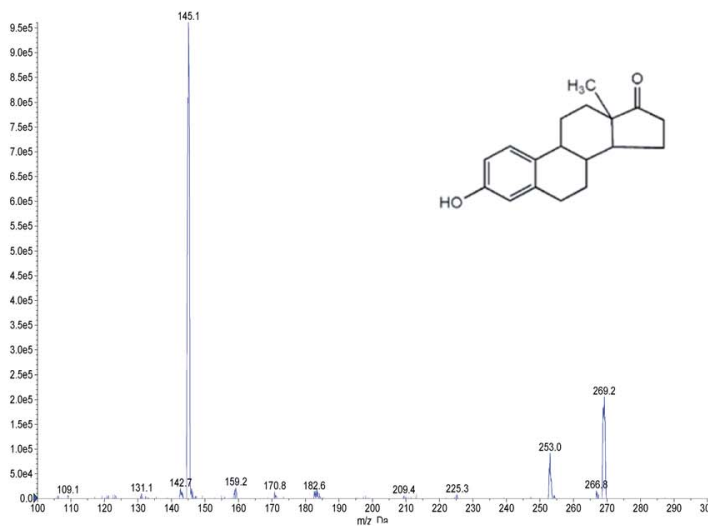

B

Fig. 1 Chemical structures and mass spectra of CuB (A) and IS (B). 
The preparation of calibration standards for the specific steps: the appropriate amount of $\mathrm{CuB}$ working solution was transferred to $1.5 \mathrm{~mL}$ centrifuge tubes, dried, and consequently mixed with $50 \mu \mathrm{L}$ aliquots of blank plasma to obtain the calibration concentrations. The resulting concentrations of $\mathrm{CuB}$ were $0.05,0.1,1.0,25,250$, and $1000 \mathrm{ng} \mathrm{mL}{ }^{-1}$. A double-blank plasma sample containing neither $\mathrm{CuB}$ nor the IS was prepared along with these calibration standards.

Quality control (QC) samples were prepared in three levels i.e. low $\left(0.1 \mathrm{ng} \mathrm{mL}{ }^{-1}\right)$, medium (25 $\left.\mathrm{ng} \mathrm{mL}{ }^{-1}\right)$ and high $\left(750 \mathrm{ng} \mathrm{mL}^{-1}\right)$ concentration of CuB. The QC samples were prepared following the method for preparation of the calibrated sample.

\subsection{Plasma sample treatment}

All plasma samples were thawed at room temperature prior to analysis. The protein precipitation procedure was applied to the sample pretreatment. Briefly, the IS solution $(50 \mu \mathrm{L}, 848 \mathrm{ng}$ $\mathrm{mL}^{-1}$ ) and $100 \mu \mathrm{L}$ of methanol were mixed with $50 \mu \mathrm{L}$ of the plasma sample in a $1.5 \mathrm{~mL}$ Eppendorf tube. The solution was then vortexed for $1.0 \mathrm{~min}$, additionally mixed with $1.0 \mathrm{~mL}$ ethyl acetate, and consequently vortexed and centrifuged at $15000 \mathrm{rpm}$ for $3 \mathrm{~min}$ and $10 \mathrm{~min}$, respectively. The supernatant fluid was then transferred to a new tube and was evaporated to dryness under a gentle nitrogen stream at $40{ }^{\circ} \mathrm{C}$. The residue was redissolved with $100 \mu \mathrm{L}$ of the mobile phase and centrifuged for $8 \mathrm{~min}$. The amount of sample injected into the UPLCMS system for analysis was $2 \mu \mathrm{L}$.

\subsection{Method validation}

The developed UPLC-MS/MS method was validated with respect to selectivity, linearity and LLOQ, matrix effects, recovery, accuracy, precision and stability according to the US Food and Drug Administration Bio-analytical Method Validation Guide. ${ }^{20}$

2.6.1. Selectivity. Blank rat plasma from six different sources was compared with the corresponding spiked plasma. Specificity was evaluated by analyzing the potential interference of endogenous substances at the retention times of $\mathrm{CuB}$ and the IS. Each blank plasma sample was detected under the LC-MS system to ensure that the plasma was free from the interference of $\mathrm{CuB}$ and the IS.

2.6.2. Linearity and LLOQ. The calibration samples were obtained by independently adding a series of IS solutions at different concentrations $\left(50 \mu \mathrm{L}, 848 \mathrm{ng} \mathrm{mL}{ }^{-1}\right)$, the working solution $(50 \mu \mathrm{L})$, and $150 \mu \mathrm{L}$ methanol to blank rat plasma (50 $\mu \mathrm{L})$. Following the procedure described above, the final concentrations ranged from 0.05 to $1000 \mathrm{ng} \mathrm{mL}{ }^{-1}$ of $\mathrm{CuB}$. The calibration curves were constructed by linear regression of the area ratio for $\mathrm{CuB} / \mathrm{IS}$ ( $Y$-axis) and concentrations ( $X$-axis) with a $1 / x$ weighting factor. Concentrations of quality controls (QCs) and samples were calculated using the regression equation of the calibration curve. The LLOQ was determined from the lowest concentration (signal-to-noise ratio $>10$ ) on the calibration curve with a precision of $\pm 20 \%$ and an accuracy of $\pm 20 \%$.

2.6.3. Matrix effects and recovery. The matrix effect was determined by comparing the peak areas of blank plasma from the spiked QC samples with those of the standard samples at equivalent concentrations (three different QC concentrations were used ( $n=6$, for each concentration)). The recovery was defined by comparing the peak areas of $\mathrm{CuB}$ extracted from plasma samples with the peak areas of the pure standard from which no extraction was performed. Each respective extraction recovery of $\mathrm{CuB}$ was measured at three different concentration levels (low, medium and high) of QC samples. The extracted samples were repeated six times at each concentration.

2.6.4. The intra-, inter-day accuracy and precision. Accuracy was described as $\mathrm{RE} \%$, and it was determined by the degree of closeness between the measured concentration and the actual concentration of the label. Relative standard deviation (RSD\%) was used to describe the precision of the determination. The QC samples were measured on the same day and after three days to calculate intraday precision and interday precision. The LLOQ was evaluated on the same day and for three consecutive validation days, with six replicates of low, medium and high concentrations of QC samples being evaluated. For the QC samples, the accuracy did not exceed 15\% RE, the precision did not exceed 15\% RSD and the LLOQ did not exceed $20 \%$.

2.6.5. Stability. The stability of $\mathrm{CuB}$ in the plasma was assessed by analyzing QC samples in rat plasma at three concentration levels, under the following four conditions: (1) long-term stability, QC samples $(n=5)$ were stored at $-80^{\circ} \mathrm{C}$ for 20 days; (2) short-term stability, QC samples $(n=5)$ were stored at room temperature for $12 \mathrm{~h}$; (3) stability to three freeze-thaw cycles, QC samples $(n=5)$ were detected after three cycles of freezing $\left(-40{ }^{\circ} \mathrm{C}\right)$ and thawing (room temperature); (4) QC samples $(n=5)$ were placed in the autosampler for $24 \mathrm{~h}$ at $4{ }^{\circ} \mathrm{C}$ after being prepared. After the four tests, the measured stability was within the allowable range of accuracy $( \pm 15 \%)$ and precision (15\% RSD).

\subsection{Pharmacokinetics study}

Twelve male Wistar rats weighing 240-280 g were subjected to two different treatments. The first group received an oral administration of $8 \mathrm{mg} \mathrm{kg}{ }^{-1} \mathrm{CuB}$ suspended in a $0.5 \% \mathrm{CMC}-\mathrm{Na}$ aqueous solution. The second group was intravenously injected with $\mathrm{CuB}$ at a dose of $1.3 \mathrm{mg} \mathrm{kg}{ }^{-1}$ dissolved in $N, N$-dimethylacetamide-PEG 400-water (2: $4: 1, \mathrm{v} / \mathrm{v} / \mathrm{v})$. After 0.083, 0.25, 0.5, $0.75,1,2,3,4,5,6,8,10,12$, and 24 hours post injection, a $0.3 \mathrm{~mL}$ blood sample was collected through the posterior orbital venous sinus and transferred to heparinized tubes. All of the samples were immediately centrifuged at $15000 \mathrm{rpm}$ for $10 \mathrm{~min}$ to separate the plasma, and then stored at $-80{ }^{\circ} \mathrm{C}$ until analysis. With the help of DAS (Drug and Statistics) software (version 3.2.8, Chinese Mathematical Pharmacology Specialized Committee, Shanghai, China), a non-compartment model was used to calculate pharmacokinetics parameters. Values were expressed as the mean \pm the standard error of the mean (SD).

\subsection{Absolute bioavailability}

The absolute bioavailability $(F)$ was calculated using the formula: ${ }^{21}$ 


$$
F(\%)=\left(\mathrm{AUC}_{\text {i.g. }}\right) \times\left(\operatorname{dose}_{\text {i.v. }}\right) /\left(\mathrm{AUC}_{\text {i.v. }}\right) \times\left(\operatorname{dose}_{\text {i.g }}\right) \times 100 \%
$$

$\mathrm{AUC}_{\text {i.g. }}$ and $\mathrm{AUC}_{\text {i.v. }}$ represented the areas under the concentration-time curves of the analytes after $\mathrm{CuB}$ was administrated orally and intravenously to the rats, respectively. Dose $e_{\text {i.g. }}$ and dose $_{\text {i.v. }}$ represented the dose of the analytes administered orally and intravenously to the rats.

\section{Results and discussion}

\subsection{Selectivity}

The selectivity was examined by analyzing blank plasma samples from six different rats. Under the LC-MS/MS conditions, no significant interference peaks from endogenous substances were observed in the retention regions of $\mathrm{CuB}$ and
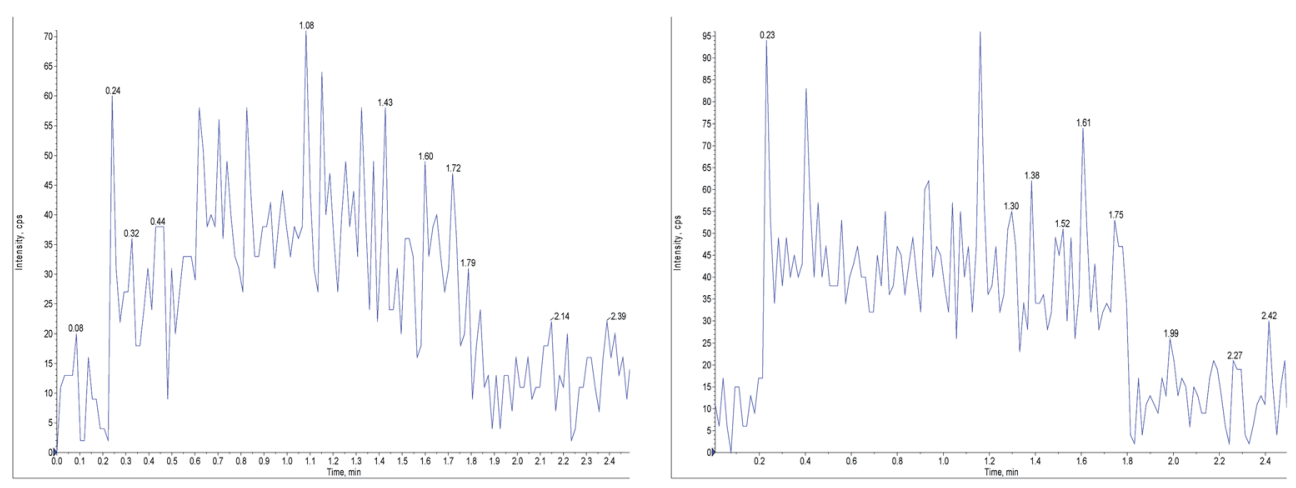

(A)
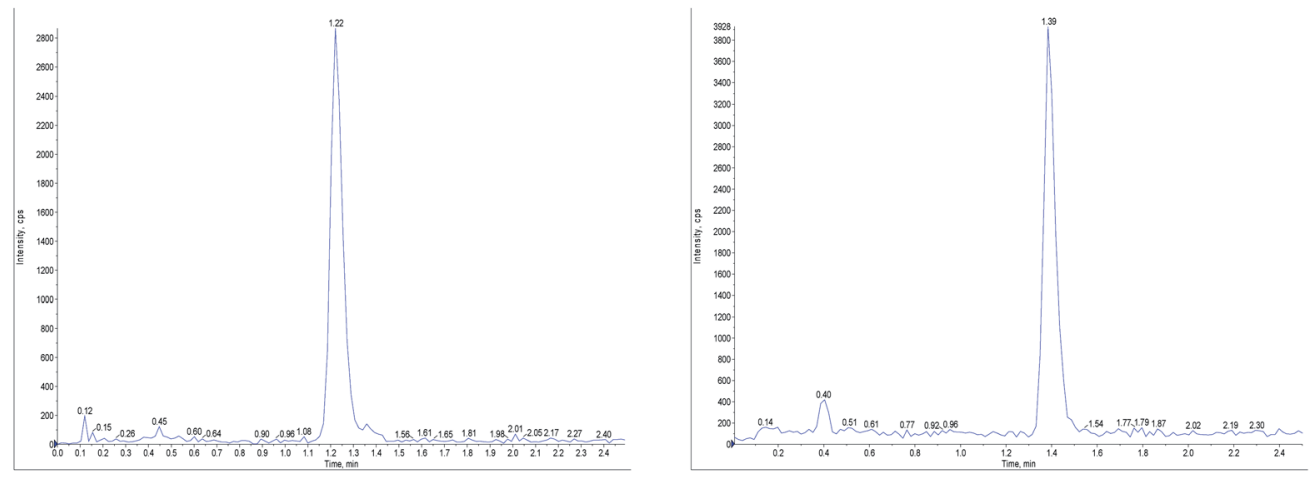

(B)
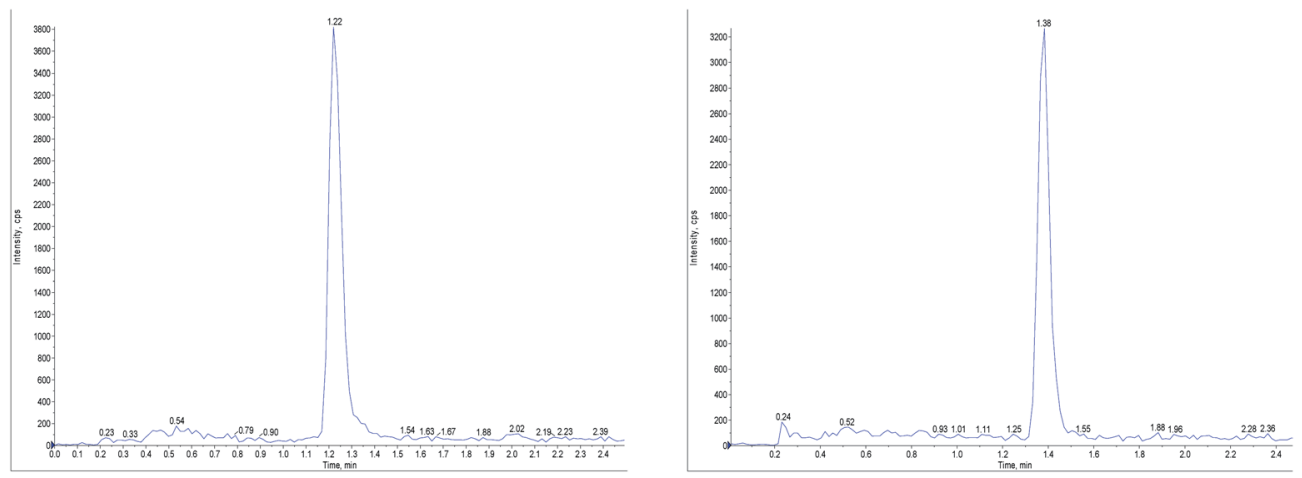

(C)

Fig. 2 Representative chromatograms of: (A) blank plasma; (B) blank plasma spiked with CuB (2 ng $\mathrm{mL}^{-1}$ for CuB) and estrone (IS); and (C) a representative plasma sample $0.5 \mathrm{~h}$ after drug administration. Left and right are the MRM scans for CuB and the IS, respectively. 
Table 1 Matrix effects and extraction recoveries of $\mathrm{CuB}$ and IS in rat plasma $(n=6)$

\begin{tabular}{lllllll}
\hline & & \multicolumn{2}{l}{ Matrix effect } & & \multicolumn{2}{l}{ Extraction recovery } \\
Ingredient & $\begin{array}{l}\text { Conc. } \\
\left(\mathrm{ng} \mathrm{mL}^{-1}\right)\end{array}$ & $\begin{array}{l}\text { Mean } \\
(\%)\end{array}$ & $\begin{array}{l}\text { RSD } \\
(\%)\end{array}$ & $\begin{array}{l}\text { Mean } \\
(\%)\end{array}$ & $\begin{array}{l}\text { RSD } \\
(\%)\end{array}$ \\
\hline \multirow{2}{*}{ CuB } & 0.1 & $96.35 \pm 3.15$ & 3.27 & $89.44 \pm 5.19$ & 6.22 \\
& 25 & $99.42 \pm 3.23$ & 3.24 & $90.53 \pm 3.65$ & 4.03 \\
& 750 & $94.19 \pm 4.14$ & 4.39 & $85.34 \pm 3.55$ & 4.16 \\
IS & 848 & $100.83 \pm 6.16$ & 5.44 & $96.38 \pm 5.82$ & 3.91
\end{tabular}

Table 2 Accuracy and precision values for the determination of CuB $(n=6)$

\begin{tabular}{|c|c|c|c|c|}
\hline \multirow[b]{2}{*}{ Conc. (ng mL $\mathrm{mL}^{-1}$ ) } & \multicolumn{2}{|c|}{ Accuracy (RE\%) } & \multicolumn{2}{|c|}{ Precision (RSD\%) } \\
\hline & Intra-day & Inter-day & Intra-day & Inter-day \\
\hline 0.05 (LLOQ) & 5.32 & 6.09 & 6.24 & 10.46 \\
\hline 0.1 & 3.17 & -2.52 & 7.89 & 8.64 \\
\hline 25 & 1.77 & 3.93 & 2.90 & 6.25 \\
\hline 750 & 3.53 & -4.03 & 1.77 & 1.68 \\
\hline
\end{tabular}

the IS. This data demonstrated the good selectivity of the LCMS/MS method. Typical chromatograms are shown in Fig. 2.

\subsection{Linearity and LLOQ}

The linear equation of the calibration curve, linear range and LLOQ of the analyte were measured by the LC-MS/MS method. The assay showed good linearity in the range of $0.05-1000 \mathrm{ng}$ $\mathrm{mL}^{-1}$ of CuB. The equation for the calibration curve was: $y=$ $16234.5 x+3365.9, r=0.9998$. This value proved that the analyte had good linearity. $x$ represented the plasma concentration while $y$ represented the ratio of the $\mathrm{CuB}$ peak area to that of the IS. The LLOQ of CuB was $0.05 \mathrm{ng} \mathrm{mL}^{-1}$. The accuracy and precision of the LLOQ were all in accordance with requirements (less than $20 \%$ ).

\subsection{Matrix effects, recovery, accuracy and precision}

Under the three QC concentration levels, the matrix effects of $\mathrm{CuB}$ in rat plasma ranged from $94.19 \%$ to $99.42 \%$, while those of the IS were $100.83 \%$. The RSD in these analyzed samples was $<7.0 \%$, which was in accordance with regulations. Therefore, no significant matrix effect was observed for the analytes. The extraction recoveries from plasma were $85.34-90.53 \%$. The mean recovery of the IS from rat plasma was $96.38 \%$ using this method. The observed data suggested that the sample treatment was suitable for the method. The data for the matrix effects and recoveries are shown in Table 1.

Accuracy and precision values were obtained by measuring the QC samples in six replicates at four concentration levels. The results from three consecutive days are shown in Table 2. The absolute values of the RE for the intra- and inter-day accuracy were $<10 \%$. The intra- and inter-day precision was below $15 \%$ with a maximum RSD of $10.46 \%$. All of the results were within a satisfactory range, which showed that the method had good operability and reproducibility. Therefore, the method was suitable for the determination of constituents in plasma samples.

\subsection{Stability}

Under the following storage conditions, the content of $\mathrm{CuB}$ in the three concentrations of rat plasma was stable. The storage conditions were as follows: preservation at $-80^{\circ} \mathrm{C}$ for 20 days, storage at room temperature for $12 \mathrm{~h}, 3$ freeze-thaw cycles or $4{ }^{\circ} \mathrm{C}$ in the autosampler stored for $24 \mathrm{~h}$. The outcomes of the stability tests are summarized in Table 3.

\subsection{Optimization of UPLC conditions}

The pretreatment of plasma samples is one of the factors that influences the determination of analytes in plasma. Due to the small sample volume, low concentration and highly interfering components of plasma samples, it is necessary to establish appropriate treatment methods to ensure the accuracy, scientificity and effectiveness of analyte determination. In the previous study, liquid-liquid extraction was used to purify components and improve sensitivity. The choice of extraction solvents is a critical step in the pretreatment of plasma samples. Extraction solvents include ethyl ether, dichloromethane, ethyl acetate and chloroform, all of which had different effects on the extraction and recovery rates of CuB. The extraction rate of ethyl acetate was significantly higher than that of the other organic solvents, without interference from endogenous substances and good reproducibility. Therefore, the liquid-liquid extraction method with ethyl acetate was finally selected for the sample pretreatment.

In order to improve the peak shape, sensitivity, and selectivity and to shorten run times, chromatographic conditions should be optimized. The mobile phases consisted of methanol-water, acetonitrile-water, methanol- $0.1 \%$ formic acid,

Table 3 Stability for the determination of the three concentrations in rat plasma $(n=5)$

\begin{tabular}{llll}
\hline & \multicolumn{1}{l}{ Accuracy/precision (mean \pm SD/RSD $\%)$} & \\
\cline { 2 - 3 } Storage conditions & 0.1 (low QC) & 25 (mid QC) & 750 (high QC) \\
\hline Long-term stability at $-80{ }^{\circ} \mathrm{C}$ for 20 days & $0.1 \pm 0.008 / 5.27$ & $24.3 \pm 0.62 / 4.85$ & $754 \pm 2.6 / 3.19$ \\
Short-term stability at room temperature for $12 \mathrm{~h}$ & $0.1 \pm 0.011 / 6.19$ & $25.7 \pm 0.38 / 5.79$ & $751 \pm 5.7 / 3.53$ \\
Freeze-thaw stability, three cycles & $0.09 \pm 0.003 / 4.78$ & $24.8 \pm 0.52 / 5.02$ & $747 \pm 4.9 / 3.91$ \\
Autosampler stability at $4{ }^{\circ} \mathrm{C}$ for $24 \mathrm{~h}$ & $0.11 \pm 0.003 / 5.71$ & $25.3 \pm 0.72 / 3.71$ & $755 \pm 2.3 / 2.96$
\end{tabular}


acetonitrile- $0.1 \%$ formic acid and other elution systems. Among these, acetonitrile- $0.1 \%$ formic acid was chosen as the best liquid phase due to producing a higher signal response and having a better peak shape than the other combinations. Finally, a good chromatographic separation was obtained under the elution conditions with acetonitrile- $0.1 \%$ formic acid at a flow rate of $0.3 \mathrm{~mL} \mathrm{~min}^{-1}$. The method has a short running time of $2.5 \mathrm{~min}$, which allows for more samples to be determined.

\subsection{Pharmacokinetics study}

The validated UPLC-MS/MS method was successfully applied for the quantitative determination of $\mathrm{CuB}$ after single-dose administration in rats. All of the measured concentrations were higher than the LLOQ in the plasma. Mean \pm SD plasma concentration-time curves of $\mathrm{CuB}$ after administration are shown in Fig. 3. The main pharmacokinetics parameters of $\mathrm{CuB}$ were identified and are listed in Table 4 . Double peaks were
Table 4 Pharmacokinetics parameters of $\mathrm{CuB}$ after administration to rats $(n=6)$

\begin{tabular}{llll}
\hline Parameters & Unit & i.g. $\left(8 \mathrm{mg} \mathrm{kg}{ }^{-1}\right)$ & i.v. $\left(1.3 \mathrm{mg} \mathrm{kg}^{-1}\right)$ \\
\hline $\mathrm{AUC}_{0-t}$ & $\mathrm{ng} \mathrm{L}^{-1} \mathrm{~h}^{-1}$ & $183.28 \pm 10.24$ & $2181.25 \pm 42.49$ \\
$\mathrm{AUC}_{0-\infty}$ & $\mathrm{ng} \mathrm{L}{ }^{-1} \mathrm{~h}^{-1}$ & $187.41 \pm 10.41$ & $2187.11 \pm 42.07$ \\
$\mathrm{MRT}_{0-t}$ & $\mathrm{~h}$ & $6.49 \pm 0.18$ & $3.65 \pm 0.068$ \\
$\mathrm{MRT}_{0-\infty}$ & $\mathrm{h}$ & $7.02 \pm 0.29$ & $3.72 \pm 0.09$ \\
$t_{1 / 2 \mathrm{z}}$ & $\mathrm{h}$ & $4.129 \pm 0.54$ & $2.77 \pm 0.28$ \\
$T_{\max }$ & $\mathrm{h}$ & 3 & $0.117 \pm 0.075$ \\
$V_{\mathrm{z}} / F$ & $\mathrm{~L} \mathrm{~kg}$ & $2.55 \times 10^{8} \pm$ & $2.38 \times 10^{6} \pm$ \\
& & $3.62 \times 10^{7}$ & $2.47 \times 10^{5}$ \\
$\mathrm{CL}_{\mathrm{z}} / F$ & $\mathrm{~L} \mathrm{~h}^{-1} \mathrm{~kg}^{-1}$ & $4.28 \times 10^{7} \pm$ & $5.95 \times 10^{5} \pm$ \\
& & $2.47 \times 10^{6}$ & $1.15 \times 10^{4}$ \\
$C_{\max }$ & $\mathrm{ng} \mathrm{L}^{-1}$ & $34.16 \pm 2.91$ & $706.55 \pm 14.58$
\end{tabular}

observed in the curves of the plasma concentration-time profiles of $\mathrm{CuB}$ after oral administration of CuB. After oral administration of $\mathrm{CuB}$ in rats, the $\mathrm{AUC}_{0-t}$ of $\mathrm{CuB}$ in rat plasma

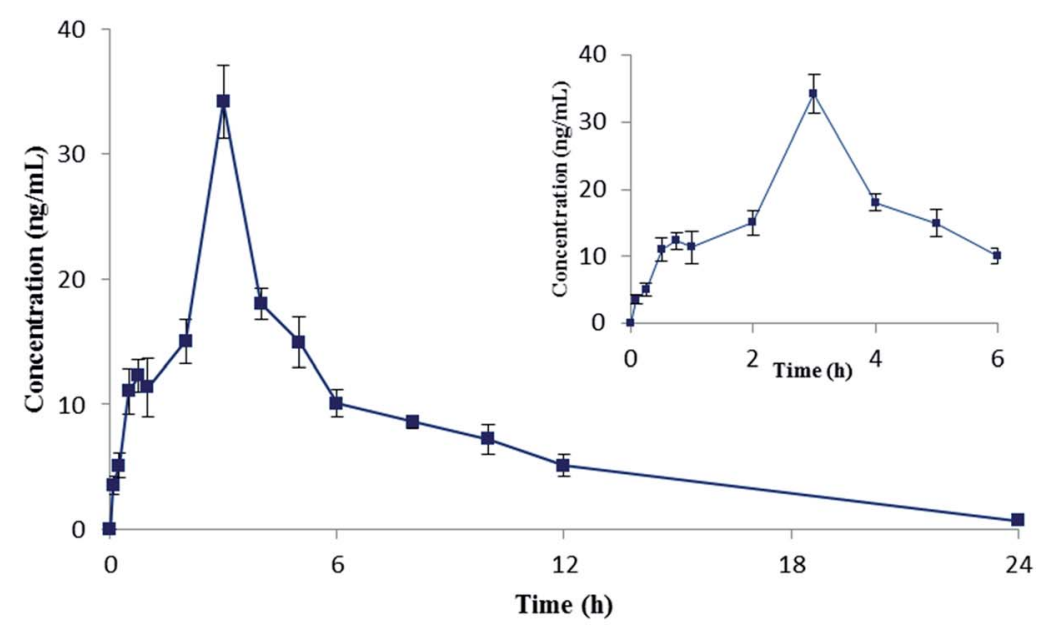

(A)

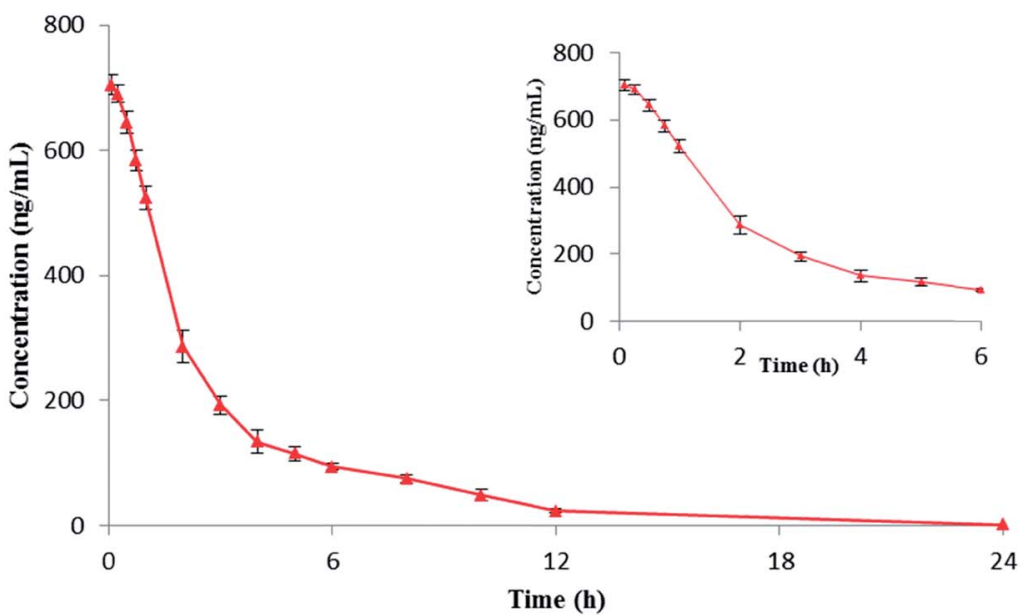

(B)

Fig. 3 Mean plasma concentration-time curves of $\mathrm{CuB}$ in rats after oral administration (A) and intravenous administration (B) of CuB. Each point represents the mean $\pm \operatorname{SD}(n=6)$. 
was $183.28 \pm 10.24 \mathrm{ng} \mathrm{h}^{-1} \mathrm{~L}^{-1} . T_{\max }, C_{\max }$, and $t_{1 / 2}$ were $3 \mathrm{~h}$, $34.16 \pm 2.91 \mathrm{ng} \mathrm{mL}^{-1}$, and $4.129 \pm 0.54 \mathrm{~h}$, respectively. Yet, the $\mathrm{AUC}_{0-t}$ of the plasma parameters of the $\mathrm{CuB}$ injection was $2181.25 \pm 42.49 \mathrm{ng} \mathrm{h}{ }^{-1} \mathrm{~L}^{-1} . T_{\max }, C_{\max }$, and $t_{1 / 2}$ were $0.117 \pm$ $0.075 \mathrm{~h}, 706.55 \pm 14.58 \mathrm{ng} \mathrm{mL}{ }^{-1}$ and $2.77 \pm 0.28 \mathrm{~h}$, respectively (Table 4). Compared with the previous methods, the approach adopted in the present article has higher experimental sensitivity and simplicity. The LLOQ was only $0.05 \mathrm{ng} \mathrm{mL} \mathrm{m}^{-1}$. In the previous study, Zhao et al. found that the elimination half-life $\left(t_{1 / 2}\right)$ of $\mathrm{CuB}$ was $2.50 \pm 0.58 \mathrm{~h},{ }^{16}$ and Wang et al. found that $\mathrm{CuB}$ in cucurbitacin tablets $t_{1 / 2}$ was $3.19 \pm 0.54 \mathrm{~h},{ }^{17}$ while the $t_{1 / 2}$ of $\mathrm{CuB}$ was $4.129 \pm 0.54 \mathrm{~h}$ in this paper. Double peaks were observed in the curves of mean plasma concentration-time profiles of $\mathrm{CuB}$ after oral administration of $\mathrm{CuB}$. This is similar to the Zhao et al. and Wang et al. studies after oral administration of CuB or cucurbitacin tablets. The reason for the double peaks phenomenon of $\mathrm{CuB}$ may be attributed to distribution reabsorption and enterohepatic circulation. It is known that the oral $\mathrm{CuB}$ has a low bioavailability problem; in the present study the absolute bioavailability of oral administration of $\mathrm{CuB}$ in rats was $1.37 \%$. The reasons leading to low absolute bioavailability are drug solubility, membrane permeability, gastrointestinal digestion and absorption. Improvement of the bioavailability is of utmost importance, and there are many ways to improve the bioavailability of oral drugs, including increasing the water solubility of drugs, inhibiting the intestinal metabolism and inhibiting the transport of intestinal mucosal carriers (such as $\mathrm{P}$ - protease inhibitors).

In a future study, we will prepare solid dispersions to improve solubility, using poloxamer as a polymeric carrier. The solid dispersion will be evaluated in vitro and in vivo using rats. The comprehensive data in our current study indicate that the method is applicable to the pharmacokinetics study of CuB in rats.

\section{Conclusions}

This study established and validated a highly selective and sensitive UPLC-MS/MS method which can be used to detect lower levels of $\mathrm{CuB}$ in rat plasma; the standard curve is over a concentration range of $0.05-1000 \mathrm{ng} \mathrm{mL}^{-1}$. The conditions for the mobile phase and sample extraction solvent were optimized. The presented method has been validated on plasma samples in experimental animals. Nevertheless, the absolute bioavailability of orally administered $\mathrm{CuB}$ in rats was only $1.37 \%$. Therefore, the absolute bioavailability of orally administered $\mathrm{CuB}$ is an urgent and challenging issue for most researchers. In addition, although the present study provided the data and theoretical basis for the application of $\mathrm{CuB}$, further studies on bioavailability are necessary.

\section{Ethical statement}

This study was performed in strict accordance with the NIH guidelines for the care and use of laboratory animals (NIH Publication No. 85-23 Rev. 1985) and was approved by the
Animal Experimental Ethics Committee of Chengde Medical College (No. CDMULAC-2017-003, Chengde, China).

\section{Conflicts of interest}

The authors declare that they have no conflicts of interest.

\section{Abbreviations}

$\begin{array}{ll}\text { CuB } & \text { Cucurbitacin B } \\ \text { IS } & \text { Internal standard } \\ \text { UPLC-MS/ } & \text { Ultra-performance liquid chromatography-mass } \\ \text { MS } & \text { spectrometry } \\ \text { LLOQ } & \text { Lower limit of quantification }\end{array}$

\section{Acknowledgements}

The study was supported by the Hebei Province Key Discipline Construction Projects of Higher School, the Science and Technology Research Youth Fund Project of Higher School in Hebei Province (No. QN2018057), and the Science and Technology Research Key Project of Chengde Medical College (No. 201710).

\section{References}

1 S. Duangmano, P. Sae-Lim, A. Suksamrarn, F. E. Domann and P. Patmasiriwat, Cucurbitacin B inhibits human breast cancer cell proliferation through disruption of microtubule polymerization and nucleophosmin/B23 translocation, BMC Complementary Altern. Med., 2012, 12, 185.

2 P. Bhandari, N. Kumar, B. Singh and V. K. Kaul, Cucurbitacins from Bacopa monnieri, Phytochemistry, 2007, 68, 1248-1254.

3 J. C. Chen, M. H. Chiu, R. L. Nie, G. A. Cordell and S. X. Qiu, Cucurbitacins and cucurbitane glycosides: structures and biological activities, Nat. Prod. Rep., 2005, 22, 386-399.

4 A. Aribi, S. Gery, D. H. Lee, N. H. Thoennissen, G. B. Thoennissen, R. Alvarez, Q. Ho, K. Lee, N. B. Doan, K. T. Chan, M. Toh, J. W. Said and H. P. Koeffler, The triterpenoid cucurbitacin $\mathrm{B}$ augments the antiproliferative activity of chemotherapy in human breast cancer, Int. J. Cancer, 2013, 132, 2730-2737.

5 K. T. Chan, K. Li, S. L. Liu, K. H. Chu, M. Toh and W. D. Xie, Cucurbitacin B inhibits STAT3 and the Raf/MEK/ERK pathway in leukemia cell line K562, Cancer Lett., 2010, 289, 46-52.

6 K. T. Chan, F. Y. Meng, Q. Li, C. Y. Ho, T. S. Lam, Y. To, W. H. Lee, M. Li, K. H. Chu and M. Toh, Cucurbitacin B induces apoptosis and S phase cell cycle arrest in BEL-7402 human hepatocellular carcinoma cells and is effective via oral administration, Cancer Lett., 2010, 294, 118-124.

7 S. Dakeng, S. Duangmano, W. Jiratchariyakul, Y. U-Pratya, O. Bögler and P. Patmasiriwat, Inhibition of Wnt signaling by cucurbitacin $\mathrm{B}$ in breast cancer cells: reduction of Wnt-associated proteins and reduced translocation of 
galectin-3-mediated $\beta$-catenin to the nucleus, J. Cell. Biochem., 2012, 113, 49-60.

8 Y. Gao, M. S. Islam, J. Tian, V. W. Lui and D. Xiao, Inactivation of ATP citrate lyase by cucurbitacin B: a bioactive compound from cucumber, inhibits prostate cancer growth, Cancer Lett., 2014, 349, 15-25.

9 X. F. Li, K. F. Liu and Y. H. Deng, Determination of the contents of cucurbitacin $\mathrm{B}$ and cucurbitacin $\mathrm{E}$ in cucurbitacin raw material by HPLC, J. Shenyang Pharm. Univ., 2010, 27, 123-125.

10 W. Sun, Z. M. Chao, C. Wang, X. Y. Wu, B. C. Yang and Z. G. Tan, Determination of cucurbitacin B in some parts of Trichosanthes kirilowii by HPLC-UV method, Chin. J. Exp. Tradit. Med. Formulae, 2014, 20, 86-88.

$11 \mathrm{~J}$. T. Dou and S. W. Li, Determination of content of cucurbitacin B in cucurbitacins by HPLC, J. Pharm. Pract., 2014, 32, 294-296.

12 N. Wang, Y. H. Deng, S. N. Wang, X. Y. Zhou, J. Zhao, L. Shen and X. H. Dong, Determination of cucurbitacin B in human serum album nanopartieles by HPLC, Chin. J. New Drugs, 2007, 16, 1968-1970.

13 N. Bajcsik, R. Pfab and J. Pietsch, Simultaneous determination of cucurbitacin B, E, I and E-glucoside in plant material and body fluids by HPLC-MS, $J$. Chromatogr. B: Anal. Technol. Biomed. Life Sci., 2017, 1052, 128-134.

14 C. Park, J. G. Ha, S. Choi, E. Kim, K. Noh, B. S. Shin and W. Kang, HPLC-MS/MS analysis of mesupron and its application to a pharmacokinetic study in rats, J. Pharm. Biomed. Anal., 2018, 150, 39-42.

15 C. Wang, X. Zhang, X. Ya, W. Ye, S. Ma and Y. Jiang, Chemical profiling of Euphorbia fischeriana Steud. by
UHPLC-Q/TOF-MS, J. Pharm. Biomed. Anal., 2018, 151, 126132.

$16 \mathrm{~W}$. Zhao, D. Xu, W. Yan, Y. Wang and N. Zhang, Development and validation of a UPLC-MS/MS method for the determination of cucurbitacin $\mathrm{B}$ in rat plasma and application to a pharmacokinetic study, Biomed. Chromatogr., 2016, 30, 503-507.

17 Z. Wang, W. Zhu, M. Gao, C. Wu, C. Yang, J. Yang, G. Wu, B. Yang and H. Kuang, Simultaneous determination of cucurbitacin $\mathrm{B}$ and cucurbitacin $\mathrm{E}$ in rat plasma by UHPLC-MS/MS: a pharmacokinetics study after oral administration of cucurbitacin tablets, J. Chromatogr. B: Anal. Technol. Biomed. Life Sci., 2017, 1065-1066, 63-69.

18 Y. Bai, Q. Zhang, B. Wang, M. Zhang, Y. Xu, S. Li, Y. Zhao and Z. Yu, Plasma pharmacokinetics, bioavailability, and tissue distribution of four C-glycosyl flavones from mung bean (Vigna radiata L.) seed extracts in rat by ultrahighperformance liquid chromatography-tandem mass spectrometry, J. Agric. Food Chem., 2017, 65(27), 5570-5580.

19 L. Z. Benet, The role of BCS (biopharmaceutics classification system) and BDDCS (biopharmaceutics drug disposition classification system) in drug development, J. Pharm. Sci., 2013, 102, 34-42.

20 US Food and Drug Administration, Guidance for Industry: Analytical Procedures and Methods Validation for Drugs and Biologics, 2015, https://www.fda.gov/Drugs.

21 Y. Bai, Q. Zhang, B. Wang, M. Zhang, Y. Xu, S. Li, Y. Zhao and Z. Yu, Plasma pharmacokinetics, bioavailability, and tissue distribution of four C-glycosyl flavones from mung mean (Vigna radiata L.) seed extracts in rat by ultrahighperformance liquid chromatography-tandem mass spectrometry, J. Agric. Food Chem., 2017, 65, 5570-5580. 